\title{
ON $\theta$-PRECONTINUOUS FUNCTIONS
}

\author{
TAKASHI NOIRI
}

(Received 16 January 2001)

\begin{abstract}
We introduce a new class of functions called $\theta$-precontinuous functions which is contained in the class of weakly precontinuous (or almost weakly continuous) functions and contains the class of almost precontinuous functions. It is shown that the $\theta$ precontinuous image of a $p$-closed space is quasi $H$-closed.
\end{abstract}

2000 Mathematics Subject Classification. 54C08.

1. Introduction. A subset $A$ of a topological space $X$ is said to be preopen [14] or nearly open [26] if $A \subset \operatorname{Int}(\mathrm{Cl}(A))$. A function $f: X \rightarrow Y$ is called precontinuous [14] if the preimage $f^{-1}(V)$ of each open set $V$ of $Y$ is preopen in $X$. Precontinuity was called near continuity by Pták [26] and also called almost continuity by Frolík [9] and Husain [10]. In 1985, Janković [12] introduced almost weak continuity as a weak form of precontinuity. Popa and Noiri [23] introduced weak precontinuity and showed that almost weak continuity is equivalent to weak precontinuity. Paul and Bhattacharyya [21] called weakly precontinuous functions quasi precontinuous and obtained the further properties of quasi precontinuity. Recently, Nasef and Noiri [16] have introduced and investigated the notion of almost precontinuity. Quite recently, Jafari and Noiri [11] investigated the further properties of almost precontinuous functions.

In this paper, we introduce a new class of functions called $\theta$-precontinuous functions which is contained in the class of weakly precontinuous functions and contains the class of almost precontinuous functions. We obtain basic properties of $\theta$ precontinuous functions. It is shown in the last section that the $\theta$-precontinuous images of $p$-closed (resp., $\beta$-connected) spaces are quasi $H$-closed (resp., semiconnected).

2. Preliminaries. Throughout, by $(X, \tau)$ and $(Y, \sigma)$ (or simply $X$ and $Y$ ) we denote topological spaces. Let $S$ be a subset of $X$. We denote the interior and the closure of $S$ by $\operatorname{Int}(S)$ and $\mathrm{Cl}(S)$, respectively. A subset $S$ is said to be preopen [14] (resp., semiopen [13], $\alpha$-open [17]) if $S \subset \operatorname{Int}(\mathrm{Cl}(S))$ (resp., $S \subset \mathrm{Cl}(\operatorname{Int}(S)), S \subset \operatorname{Int}(\mathrm{Cl}(\operatorname{Int}(S))))$. The complement of a preopen set is called preclosed. The intersection of all preclosed sets containing $S$ is called the preclosure [8] of $S$ and is denoted by $\operatorname{pCl}(S)$. The preinterior of $S$ is defined by the union of all preopen sets contained in $S$ and is denoted by $\operatorname{pInt}(S)$. The family of all preopen sets of $X$ is denoted by $\mathrm{PO}(X)$. We set $\mathrm{PO}(X, x)=\{U$ : $x \in U$ and $U \in \mathrm{PO}(X)\}$. A point $x$ of $X$ is called a $\theta$-cluster point of $S$ if $\operatorname{Cl}(U) \cap S \neq \varnothing$ for every open set $U$ of $X$ containing $x$. The set of all $\theta$-cluster points of $S$ is called the $\theta$-closure of $S$ and is denoted by $\mathrm{Cl}_{\theta}(S)$. A subset $S$ is said to be $\theta$-closed [27] if $S=\mathrm{Cl}_{\theta}(S)$. The complement of a $\theta$-closed set is said to be $\theta$-open. A point $x$ of $X$ 
is called a pre $\theta$-cluster point of $S$ if $\operatorname{pCl}(U) \cap S \neq \varnothing$ for every preopen set $U$ of $X$ containing $x$. The set of all pre $\theta$-cluster points of $S$ is called the pre $\theta$-closure of $S$ and is denoted by $\mathrm{pCl}_{\theta}(S)$. A subset $S$ is said to be pre $\theta$-closed [20] if $S=\mathrm{pCl}_{\theta}(S)$. The complement of a pre $\theta$-closed set is said to be pre $\theta$-open.

Definition 2.1. A function $f: X \rightarrow Y$ is said to be precontinuous [14] (resp., almost precontinuous [16], weakly precontinuous [23] or quasi precontinuous [21]) if for each $x \in X$ and each open set $V$ of $Y$ containing $f(x)$, there exists $U \in \operatorname{PO}(X, x)$ such that $f(U) \subset V$ (resp., $f(U) \subset \operatorname{Int}(\mathrm{Cl}(V)), f(U) \subset \mathrm{Cl}(V))$.

DeFinITION 2.2. A function $f: X \rightarrow Y$ is said to be almost weakly continuous [12] if $f^{-1}(V) \subset \operatorname{Int}\left(\mathrm{Cl}\left(f^{-1}(\mathrm{Cl}(V))\right)\right)$ for every open set $V$ of $Y$.

DEFINITION 2.3. A function $f: X \rightarrow Y$ is said to be strongly $\theta$-precontinuous [19] if for each $x \in X$ and each open set $V$ of $Y$ containing $f(x)$, there exists $U \in \operatorname{PO}(X, x)$ such that $f(\operatorname{pCl}(U)) \subset V$.

DEFINITION 2.4. A function $f: X \rightarrow Y$ is said to be $\theta$-precontinuous if for each $x \in X$ and each open set $V$ of $Y$ containing $f(x)$, there exists $U \in \operatorname{PO}(X, x)$ such that $f(\operatorname{pCl}(U)) \subset \mathrm{Cl}(V)$.

REMARK 2.5. By the above definitions and Theorem 3.3 below, we have the following implications and none of these implications is reversible by [19, Example 2.2], [11, Example 2.9], and Examples 2.6 and 5.11 below.

$$
\begin{aligned}
\text { strongly } \theta \text {-precontinuous } & \Longrightarrow \text { precontinuous } \Longrightarrow \text { almost precontinuous } \\
& \Longrightarrow \theta \text {-precontinuous } \Longrightarrow \text { weakly precontinuous. }
\end{aligned}
$$

EXAMPLE 2.6. This example is due to Arya and Deb [4]. Let $X$ be the set of all real numbers. The topology $\tau$ on $X$ is the cocountable topology. Let $Y=\{a, b, c\}$, $\sigma=\{\varnothing, Y,\{a\},\{c\},\{a, c\}\}$. We define a function $f:(X, \tau) \rightarrow(Y, \sigma)$ by $f(x)=a$ if $x$ is rational; $f(x)=b$ if $x$ is irrational. Then $f$ is a $\theta$-precontinuous function which is not almost precontinuous

\section{Characterizations}

THEOREM 3.1. For a function $f: X \rightarrow Y$ the following properties are equivalent:

(1) $f$ is $\theta$-precontinuous;

(2) $\mathrm{pCl}_{\theta}\left(f^{-1}(B)\right) \subset f^{-1}\left(\mathrm{Cl}_{\theta}(B)\right)$ for every subset $B$ of $Y$;

(3) $f\left(\mathrm{pCl}_{\theta}(A)\right) \subset \mathrm{Cl}_{\theta}(f(A))$ for every subset $A$ of $X$.

Proof. $(1) \Rightarrow(2)$. Let $B$ be any subset of $Y$. Suppose that $x \notin f^{-1}\left(\mathrm{Cl}_{\theta}(B)\right)$. Then $f(x) \notin \mathrm{Cl}_{\theta}(B)$ and there exists an open set $V$ containing $f(x)$ such that $\mathrm{Cl}(V) \cap B=\varnothing$. Since $f$ is $\theta$.p.c., there exists $U \in \mathrm{PO}(X, x)$ such that $f(\mathrm{pCl}(U)) \subset \mathrm{Cl}(V)$. Therefore, we have $f(\mathrm{pCl}(U)) \cap B=\varnothing$ and $\mathrm{pCl}(U) \cap f^{-1}(B)=\varnothing$. This shows that $x \notin \mathrm{pCl}_{\theta}\left(f^{-1}(B)\right)$. Thus, we obtain $\mathrm{pCl}_{\theta}\left(f^{-1}(B)\right) \subset f^{-1}\left(\mathrm{Cl}_{\theta}(B)\right)$. 
(2) $\Rightarrow(3)$. Let $A$ be any subset of $X$. Then we have $\mathrm{pCl}_{\theta}(A) \subset \mathrm{pCl}_{\theta}\left(f^{-1}(f(A))\right) \subset$ $f^{-1}\left(\mathrm{Cl}_{\theta}(f(A))\right)$ and hence $f\left(\mathrm{pCl}_{\theta}(A)\right) \subset \mathrm{Cl}_{\theta}(f(A))$.

(3) $\Rightarrow(2)$. Let $B$ be a subset of $Y$. We have $f\left(\mathrm{pCl}_{\theta}\left(f^{-1}(B)\right)\right) \subset \mathrm{Cl}_{\theta}\left(f\left(f^{-1}(B)\right)\right) \subset \mathrm{Cl}_{\theta}(B)$ and hence $\mathrm{pCl}_{\theta}\left(f^{-1}(B)\right) \subset f^{-1}\left(\mathrm{Cl}_{\theta}(B)\right)$.

(2) $\Rightarrow(1)$. Let $x \in X$ and $V$ be an open set of $Y$ containing $f(x)$. Then we have $\mathrm{Cl}(V) \cap$ $(Y-\mathrm{Cl}(V))=\varnothing$ and $f(x) \notin \mathrm{Cl}_{\theta}(Y-\mathrm{Cl}(V))$. Hence, $x \notin f^{-1}\left(\mathrm{Cl}_{\theta}(Y-\mathrm{Cl}(V))\right)$ and $x \notin$ $\mathrm{pCl}_{\theta}\left(f^{-1}(Y-\mathrm{Cl}(V))\right)$. There exists $U \in \mathrm{PO}(X, x)$ such that $\mathrm{pCl}(U) \cap f^{-1}(Y-\mathrm{Cl}(V))=$ $\varnothing$; hence $f(\operatorname{pCl}(U)) \subset \mathrm{Cl}(V)$. Therefore, $f$ is $\theta$.p.c.

THEOREM 3.2. For a function $f: X \rightarrow Y$ the following properties are equivalent:

(1) $f$ is $\theta$-precontinuous;

(2) $f^{-1}(V) \subset \operatorname{pInt}_{\theta}\left(f^{-1}(\mathrm{Cl}(V))\right)$ for every open set $V$ of $Y$;

(3) $\mathrm{pCl}_{\theta}\left(f^{-1}(V)\right) \subset f^{-1}(\mathrm{Cl}(V))$ for every open set $V$ of $Y$.

Proof. $(1) \Rightarrow(2)$. Suppose that $V$ is any open set of $Y$ and $x \in f^{-1}(V)$. Then $f(x) \in$ $V$ and there exists $U \in \mathrm{PO}(X, x)$ such that $f(\operatorname{pCl}(U)) \subset \mathrm{Cl}(V)$. Therefore, $x \in U \subset$ $\operatorname{pCl}(U) \subset f^{-1}(\mathrm{Cl}(V))$. This shows that $x \in \operatorname{pInt}_{\theta}\left(f^{-1}(\mathrm{Cl}(V))\right)$. Therefore, we obtain $f^{-1}(V) \subset \operatorname{pInt}_{\theta}\left(f^{-1}(\mathrm{Cl}(V))\right)$.

(2) $\Rightarrow(3)$. Suppose that $V$ is any open set of $Y$ and $x \notin f^{-1}(\mathrm{Cl}(V))$. Then $f(x) \notin$ $\mathrm{Cl}(V)$ and there exists an open set $W$ containing $f(x)$ such that $W \cap V=\varnothing$; hence $\mathrm{Cl}(W) \cap V=\varnothing$. Therefore, we have $f^{-1}(\mathrm{Cl}(W)) \cap f^{-1}(V)=\varnothing$. Since $x \in f^{-1}(W)$, by (2) $x \in \operatorname{pInt}_{\theta}\left(f^{-1}(\mathrm{Cl}(W))\right)$. There exists $U \in \mathrm{PO}(X, x)$ such that $\mathrm{pCl}(U) \subset f^{-1}(\mathrm{Cl}(W))$. Thus we have $\operatorname{pCl}(U) \cap f^{-1}(V)=\varnothing$ and hence $x \notin \mathrm{pCl}_{\theta}\left(f^{-1}(V)\right)$. This shows that $\mathrm{pCl}_{\theta}\left(f^{-1}(V)\right) \subset f^{-1}(\mathrm{Cl}(V))$.

(3) $\Rightarrow(1)$. Suppose that $x \in X$ and $V$ is any open set of $Y$ containing $f(x)$. Then $V \cap(Y-\mathrm{Cl}(V))=\varnothing$ and $f(x) \notin \mathrm{Cl}(Y-\mathrm{Cl}(V))$. Therefore, $x \notin f^{-1}(\mathrm{Cl}(Y-\mathrm{Cl}(V)))$ and by (3) $x \notin \mathrm{pCl}_{\theta}\left(f^{-1}(Y-\mathrm{Cl}(V))\right)$. There exists $U \in \mathrm{PO}(X, x)$ such that $\mathrm{pCl}(U) \cap f^{-1}(Y-$ $\mathrm{Cl}(V))=\varnothing$. Therefore, we obtain $f(\mathrm{pCl}(U)) \subset \mathrm{Cl}(V)$. This shows that $f$ is $\theta$.p.c.

THEOREM 3.3. For a function $f: X \rightarrow Y$ the following properties hold:

(1) if $f$ is almost precontinuous, then it is $\theta$-precontinuous;

(2) if $f$ is $\theta$-precontinuous, then it is weakly precontinuous.

Proof. Statement (2) is obvious. We will show statement (1). Suppose that $x \in$ $X$ and $V$ is any open set of $Y$ containing $f(x)$. Since $f$ is almost precontinuous, $f^{-1}(\operatorname{Int}(\mathrm{Cl}(V)))$ is preopen and $f^{-1}(\mathrm{Cl}(V))$ is preclosed in $X$ by [16, Theorem 3.1]. Now, set $U=f^{-1}(\operatorname{Int}(\mathrm{Cl}(V)))$. Then we have $U \in \operatorname{PO}(X, x)$ and $\operatorname{pCl}(U) \subset f^{-1}(\mathrm{Cl}(V))$. Therefore, we obtain $f(\mathrm{pCl}(U)) \subset \mathrm{Cl}(V)$. This shows that $f$ is $\theta$.p.c.

COROLLARY 3.4. Let $Y$ be a regular space. Then, for a function $f: X \rightarrow Y$ the following properties are equivalent:

(1) $f$ is strongly $\theta$-precontinuous;

(2) $f$ is precontinuous;

(3) $f$ is almost precontinuous;

(4) $f$ is $\theta$-precontinuous;

(5) $f$ is weakly precontinuous.

Proof. This is an immediate consequence of [19, Theorem 3.2]. 
DEFINITION 3.5. A topological space $X$ is said to be pre-regular [20] if for each preclosed set $F$ and each point $x \in X-F$, there exist disjoint preopen sets $U$ and $V$ such that $x \in U$ and $F \subset V$.

LEMMA 3.6 (see [20]). A topological space $X$ is pre-regular if and only if for each $U \in$ $\mathrm{PO}(X)$ and each point $x \in U$, there exists $V \in \mathrm{PO}(X, x)$ such that $x \in V \subset \mathrm{pCl}(V) \subset U$.

THEOREM 3.7. Let $X$ be a pre-regular space. Then $f: X \rightarrow Y$ is $\theta . p . c$. if and only if it is weakly precontinuous.

Proof. Suppose that $f$ is weakly precontinuous. Let $x \in X$ and $V$ is any open set of $Y$ containing $f(x)$. Then, there exists $U \in \mathrm{PO}(X, x)$ such that $f(U) \subset \mathrm{Cl}(V)$. Since $X$ is pre-regular, there exists $U_{*} \in \mathrm{PO}(X, x)$ such that $x \in U_{*} \subset \mathrm{pCl}\left(U_{*}\right) \subset U$. Therefore, we obtain $f\left(\operatorname{pCl}\left(U_{*}\right)\right) \subset \mathrm{Cl}(V)$. This shows that $f$ is $\theta$.p.c.

THEOREM 3.8. Let $f: X \rightarrow Y$ be a function and $g: X \rightarrow X \times Y$ the graph function of $f$ defined by $g(x)=(x, f(x))$ for each $x \in X$. Then $g$ is $\theta$.p.c. if and only if $f$ is $\theta$.p.c.

\section{PROOF}

NeCESSITY. Suppose that $g$ is $\theta . p . c$. Let $x \in X$ and $V$ be an open set of $Y$ containing $f(x)$. Then $X \times V$ is an open set of $X \times Y$ containing $g(x)$. Since $g$ is $\theta . p . c$., there exists $U \in \mathrm{PO}(X, x)$ such that $g(\mathrm{pCl}(U)) \subset \mathrm{Cl}(X \times V)$. It follows that $\mathrm{Cl}(X \times V)=X \times \mathrm{Cl}(V)$. Therefore, we obtain $f(\mathrm{pCl}(U)) \subset \mathrm{Cl}(V)$. This shows that $f$ is $\theta$.p.c.

SuFfiCIENCY. Let $x \in X$ and $W$ be any open set of $X \times Y$ containing $g(x)$. There exist open sets $U_{1} \subset X$ and $V \subset Y$ such that $g(x)=(x, f(x)) \in U_{1} \times V \subset W$. Since $f$ is $\theta . p . c$., there exists $U_{2} \in \mathrm{PO}(X, x)$ such that $f\left(\mathrm{pCl}\left(U_{2}\right)\right) \subset \mathrm{Cl}(V)$. Let $U=U_{1} \cap U_{2}$, then $U \in \mathrm{PO}(X, x)$. Therefore, we obtain $g(\operatorname{pCl}(U)) \subset \mathrm{Cl}\left(U_{1}\right) \times f\left(\operatorname{pCl}\left(U_{2}\right)\right) \subset \mathrm{Cl}\left(U_{1}\right) \times$ $\mathrm{Cl}(V) \subset \mathrm{Cl}(W)$. This shows that $g$ is A.p.c.

\section{Some properties}

LEMMA 4.1 (see [15]). Let $A$ and $X_{0}$ be subsets of a space $X$.

(1) If $A \in \mathrm{PO}(X)$ and $X_{0}$ is semi-open in $X$, then $A \cap X_{0} \in \mathrm{PO}\left(X_{0}\right)$.

(2) If $A \in \mathrm{PO}\left(X_{0}\right)$ and $X_{0} \in \mathrm{PO}(X)$, then $A \in \mathrm{PO}(X)$.

LemmA 4.2 (see [7]). Let $A$ and $X_{0}$ be subsets of a space $X$ such that $A \subset X_{0} \subset X$. Let $\mathrm{pCl}_{X_{0}}(A)$ denote the preclosure of $A$ in the subspace $X_{0}$.

(1) If $X_{0}$ is semi-open in $X$, then $\mathrm{pCl}_{X_{0}}(A) \subset \mathrm{pCl}(A)$.

(2) If $A \in \mathrm{PO}\left(X_{0}\right)$ and $X_{0} \in \mathrm{PO}(X)$, then $\mathrm{pCl}(A) \subset \mathrm{pCl}_{X_{0}}(A)$.

THEOREM 4.3. If $f: X \rightarrow Y$ is $\theta$.p.c. and $X_{0}$ is a semi-open subset of $X$, then the restriction $f / X_{0}: X_{0} \rightarrow Y$ is $\theta$.p.c.

Proof. For any $x \in X_{0}$ and any open neighborhood $V$ of $f(x)$, there exists $U \in$ $\mathrm{PO}(X, x)$ such that $f(\operatorname{pCl}(U)) \subset \mathrm{Cl}(V)$ since $f$ is $\theta$.p.c. Put $U_{0}=U \cap X_{0}$, then by Lemmas 4.1 and $4.2 U_{0} \in \mathrm{PO}\left(X_{0}, x\right)$ and $\mathrm{pCl}_{X_{0}}\left(U_{0}\right) \subset \mathrm{pCl}\left(U_{0}\right)$. Therefore, we obtain

$$
\left(f / X_{0}\right)\left(\mathrm{pCl}_{X_{0}}\left(U_{0}\right)\right)=f\left(\mathrm{pCl}_{X_{0}}\left(U_{0}\right)\right) \subset f\left(\operatorname{pCl}\left(U_{0}\right)\right) \subset f(\operatorname{pCl}(U)) \subset \mathrm{Cl}(V) .
$$

This shows that $f / X_{0}$ is $\theta . p . c$. 
TheOREM 4.4. A function $f: X \rightarrow Y$ is $\theta$.p.c. if for each $x \in X$ there exists $X_{0} \in$ $\mathrm{PO}(X, x)$ such that the restriction $f / X_{0}: X_{0} \rightarrow Y$ is $\theta . p . c$.

Proof. Let $x \in X$ and $V$ be any open neighborhood of $f(x)$. There exists $X_{0} \in$ $\mathrm{PO}(X, x)$ such that $f / X_{0}: X_{0} \rightarrow Y$ is $\theta . p . c$. Thus, there exists $U \in \operatorname{PO}\left(X_{0}, x\right)$ such that $\left(f / X_{0}\right)\left(\mathrm{pCl}_{X_{0}}(U)\right) \subset \mathrm{Cl}(V)$. By Lemmas 4.1 and $4.2, U \in \mathrm{PO}(X, x)$ and $\mathrm{pCl}(U) \subset$ $\mathrm{pCl}_{X_{0}}(U)$. Hence, we have $f(\mathrm{pCl}(U))=\left(f / X_{0}\right)(\mathrm{pCl}(U)) \subset\left(f / X_{0}\right)\left(\mathrm{pCl}_{X_{0}}(U)\right) \subset \mathrm{Cl}(V)$. This shows that $f$ is $\theta . p . c$.

Corollary 4.5. Let $\left\{U_{\lambda}: \lambda \in \Lambda\right\}$ be an $\alpha$-open cover of a topological space $X$. A function $f: X \rightarrow Y$ is $\theta$.p.c. if and only if the restriction $f / U_{\lambda}: U_{\lambda} \rightarrow Y$ is $\theta$.p.c. for each $\lambda \in \Lambda$.

Proof. This is an immediate consequence of Theorems 4.3 and 4.4.

Let $\left\{X_{\alpha}: \alpha \in \mathscr{A}\right\}$ be a family of topological spaces, $A_{\alpha}$ a nonempty subset of $X_{\alpha}$ for each $\alpha \in \mathscr{A}$ and $X=\Pi\left\{X_{\alpha}: \alpha \in \mathscr{A}\right\}$ denote the product space, where $\mathscr{A}$ is nonempty.

LEMMA 4.6 (see [8]). Let $n$ be a positive integer and $A=\prod_{j=1}^{n} A_{\alpha_{j}} \times \Pi_{\alpha \neq \alpha_{j}} X_{\alpha}$.

(1) $A \in \mathrm{PO}(X)$ if and only if $A_{\alpha_{j}} \in \mathrm{PO}\left(X_{\alpha_{j}}\right)$ for each $j=1,2, \ldots, n$.

(2) $\mathrm{pCl}\left(\Pi_{\alpha \in \mathscr{A}} A_{\alpha}\right) \subset \Pi_{\alpha \in \mathscr{A}} \mathrm{pCl}\left(A_{\alpha}\right)$.

THEOREM 4.7. If a function $f_{\alpha}: X_{\alpha} \rightarrow Y_{\alpha}$ is $\theta$.p.c. for each $\alpha \in \mathscr{A}$. Then the product function $f: \Pi X_{\alpha} \rightarrow \Pi Y_{\alpha}$, defined by $f\left(\left\{x_{\alpha}\right\}\right)=\left\{f_{\alpha}\left(x_{\alpha}\right)\right\}$ for each $x=\left\{x_{\alpha}\right\}$, is $\theta$.p.c.

Proof. Let $x=\left\{x_{\alpha}\right\} \in \Pi X_{\alpha}$ and $W$ be any open set of $\Pi Y_{\alpha}$ containing $f(x)$. Then, there exists an open set $V_{\alpha_{j}}$ of $Y_{\alpha_{j}}$ such that

$$
f(x)=\left\{f_{\alpha}\left(x_{\alpha}\right)\right\} \in \Pi_{j=1}^{n} V_{\alpha_{j}} \times \Pi_{\alpha \neq \alpha_{j}} Y_{\alpha} \subset W .
$$

Since $f_{\alpha}$ is $\theta$.p.c. for each $\alpha$, there exists $U_{\alpha_{j}} \in \operatorname{PO}\left(X_{\alpha_{j}}, x_{\alpha_{j}}\right)$ such that $f_{\alpha_{j}}\left(\operatorname{pCl}\left(U_{\alpha_{j}}\right)\right)$ $\subset \mathrm{Cl}\left(V_{\alpha_{j}}\right)$ for $j=1,2, \ldots, n$. Now, put $U=\prod_{j=1}^{n} U_{\alpha_{j}} \times \prod_{\alpha \neq \alpha_{j}} X_{\alpha}$. Then, it follows from Lemma 4.6 that $U \in \mathrm{PO}\left(\Pi X_{\alpha}, x\right)$. Moreover, we have

$$
\begin{aligned}
f(\operatorname{pCl}(U)) & \subset f\left(\Pi_{j=1}^{n} \operatorname{pCl}\left(U_{\alpha_{j}}\right) \times \Pi_{\alpha \neq \alpha_{j}} X_{\alpha}\right) \\
& \subset \Pi_{j=1}^{n} f_{\alpha_{j}}\left(\operatorname{pCl}\left(U_{\alpha_{j}}\right)\right) \times \Pi_{\alpha \neq \alpha_{j}} Y_{\alpha} \\
& \subset \Pi_{j=1}^{n} \operatorname{Cl}\left(V_{\alpha_{j}}\right) \times \Pi_{\alpha \neq \alpha_{j}} Y_{\alpha} \subset \mathrm{Cl}(W) .
\end{aligned}
$$

This shows that $f$ is $\theta . p . c$.

\section{Preservation property}

Definition 5.1. A topological space $X$ is said to be

(1) $p$-closed [7] (resp., $p$-Lindelöf) if every cover of $X$ by preopen sets has a finite (resp., countable) subfamily whose preclosures cover $X$,

(2) countably $p$-closed if every countable cover of $X$ by preopen sets has a finite subfamily whose preclosures cover $X$;

(3) quasi H-closed [25] (resp., almost Lindelöf [6]) if every cover of $X$ by open sets has a finite (resp., countable) subfamily whose closures cover $X$,

(4) lightly compact [5] if every countable cover of $X$ by open sets has a finite subfamily whose closures cover $X$. 
DeFinition 5.2. A subset $K$ of a space $X$ is said to be

(1) $p$-closed relative to $X$ [7] if for every cover $\left\{V_{\alpha}: \alpha \in \mathscr{A}\right\}$ of $K$ by preopen sets of $X$, there exists a finite subset $\mathscr{A}_{*}$ of $\mathscr{A}$ such that $K \subset \cup\left\{\operatorname{pCl}\left(V_{\alpha}\right): \alpha \in \mathscr{A}_{*}\right\}$,

(2) quasi $H$-closed relative to $X$ [25] if for every cover $\left\{V_{\alpha}: \alpha \in \mathscr{A}\right\}$ of $K$ by open sets of $X$, there exists a finite subset $\mathscr{A}_{*}$ of $\mathscr{A}$ such that $K \subset \cup\left\{\mathrm{Cl}\left(V_{\alpha}\right): \alpha \in \mathscr{A}_{*}\right\}$.

THEOREM 5.3. If $f: X \rightarrow Y$ is a $\theta$.p.c. function and $K$ is $p$-closed relative to $X$, then $f(K)$ is quasi $H$-closed relative to $Y$.

Proof. Suppose that $f: X \rightarrow Y$ is $\theta . p$.c. and $K$ is $p$-closed relative to $X$. Let $\left\{V_{\alpha}\right.$ : $\alpha \in \mathscr{A}\}$ be a cover of $f(K)$ by open sets of $Y$. For each point $x \in K$, there exists $\alpha(x) \in \mathscr{A}$ such that $f(x) \in V_{\alpha(x)}$. Since $f$ is $\theta . p . c$., there exists $U_{x} \in \operatorname{PO}(X, x)$ such that $f\left(\operatorname{pCl}\left(U_{x}\right)\right) \subset \mathrm{Cl}\left(V_{\alpha(x)}\right)$. The family $\left\{U_{x}: x \in K\right\}$ is a cover of $K$ by preopen sets of $X$ and hence there exists a finite subset $K_{*}$ of $K$ such that $K \subset \cup_{x \in K_{*}} \operatorname{pCl}\left(U_{x}\right)$. Therefore, we obtain $f(K) \subset \cup_{x \in K *} \mathrm{Cl}\left(V_{\alpha(x)}\right)$. This shows that $f(K)$ is quasi $H$-closed relative to $Y$.

COROLlary 5.4. Let $f: X \rightarrow Y$ be a $\theta$.p.c. surjection. Then, the following properties hold:

(1) If $X$ is $p$-closed, then $Y$ is quasi $H$-closed.

(2) If $X$ is $p$-Lindelöf, then $Y$ is almost Lindelöf.

(3) If $X$ is countably $p$-closed, then $Y$ is lightly compact.

A subset $S$ of a topological space $X$ is said to be $\beta$-open [1] or semipreopen [3] if $S \subset \mathrm{Cl}(\operatorname{Int}(\mathrm{Cl}(S)))$. It is well known that $\alpha$-openness implies both preopenness and semi-openness which imply $\beta$-openness. The complement of a semipreopen set is said to be semipreclosed [3]. The intersection of all semipreclosed sets of $X$ containing a subset $S$ is the semipreclosure of $S$ and is denoted by $\operatorname{spCl}(S)$ [3].

DEFINITION 5.5. A topological space $X$ is said to be

(1) $\beta$-connected [24] or semipreconnected [2] if $X$ cannot be expressed as the union of two nonempty disjoint $\beta$-open sets,

(2) semi-connected [22] if $X$ cannot be expressed as the union of two nonempty disjoint semi-open sets.

REMARK 5.6. We have the following implications:

$$
\beta \text {-connected } \Longrightarrow \text { semi-connected } \Longrightarrow \text { connected. }
$$

But, the converses need not be true as the following simple examples show.

EXAMPLE 5.7. (1) Let $X=\{a, b, c\}$ and $\tau=\{X, \varnothing,\{a\},\{b\},\{a, b\}\}$. Then $(X, \tau)$ is connected but not semi-connected.

(2) Let $X=\{a, b, c\}$ and $\tau=\{X, \varnothing,\{b, c\}\}$. Then $(X, \tau)$ is semi-connected but not $\beta$-connected.

LEMMA 5.8. For a topological space $X$, the following properties are equivalent:

(1) $X$ is $\beta$-connected or semipreconnected.

(2) The intersection of two nonempty semipreopen subsets of $X$ is always nonempty.

(3) The intersection of two nonempty preopen subsets of $X$ is always nonempty. 
(4) $\mathrm{pCl}(V)=X$ for every nonempty preopen subset $V$ of $X$.

(5) $\operatorname{spCl}(V)=X$ for every nonempty semipreopen subset $V$ of $X$.

Proof. The proofs of equivalences of (1), (2), and (3) are given in [2, Theorem 6.4]. The other properties (4) and (5), which are stated in [18], are easily equivalent to (3) and (2), respectively.

THEOREM 5.9. If $f: X \rightarrow Y$ is a $\theta$.p.c. surjection and $X$ is $\beta$-connected, then $Y$ is semi-connected.

Proof. Let $V$ be any nonempty open set of $Y$. Let $y \in V$. Since $f$ is surjective, there exists $x \in X$ such that $f(x)=y$. Since $f$ is $\theta$.p.c., there exists $U \in \operatorname{PO}(X, x)$ such that $f(\mathrm{pCl}(U)) \subset \mathrm{Cl}(V)$. Since $X$ is $\beta$-connected, by Lemma $5.8 \mathrm{pCl}(U)=X$ and hence $\mathrm{Cl}(V)=Y$ since $f$ is surjective. Therefore, it follows from [22, Theorem 4.3] that $Y$ is semi-connected.

REMARK 5.10. The following example shows that the image of $\beta$-connectedness under weakly precontinuous surjections is not necessarily semi-connected.

EXAMPLE 5.11. Let $X$ be the set of real numbers, $\tau=\{\varnothing\} \cup\{V \subset X: 0 \in V\}$, $Y=\{a, b, c\}$, and $\sigma=\{Y, \varnothing,\{a\},\{b\},\{a, b\}\}$. Define a function $f:(X, \tau) \rightarrow(Y, \sigma)$ as follows: $f(x)=a$ if $x<0 ; f(x)=c$ if $x=0$; $f(x)=b$ if $x>0$. Then $f$ is a weakly precontinuous surjection which is not $\theta$.p.c. The topological space $(X, \tau)$ is $\beta$-connected by Lemma 5.8. By Example 5.7(1), $(Y, \sigma)$ is connected but not semi-connected.

\section{REFERENCES}

[1] M. E. Abd El-Monsef, S. N. El-Deeb, and R. A. Mahmoud, $\beta$-open sets and $\beta$-continuous mapping, Bull. Fac. Sci. Assiut Univ. A 12 (1983), no. 1, 77-90. MR 87b:54002. Zbl 577.54008.

[2] T. Aho and T. Nieminen, Spaces in which preopen subsets are semiopen, Ricerche Mat. 43 (1994), no. 1, 45-59. MR 96b:54058. Zbl 912.54030.

[3] D. Andrijević, Semipreopen sets, Mat. Vesnik 38 (1986), no. 1, 24-32. MR 87j:54002. Zbl 604.54002.

[4] S. P. Arya and M. Deb, On 8-continuous mappings, Math. Student 42 (1974), 81-89. MR 53\#3982.

[5] R. W. Bagley, E. H. Connell, and J. D. McKnight Jr., On properties characterizing pseudo-compact spaces, Proc. Amer. Math. Soc. 9 (1958), 500-506. MR $20 \# 3523$. Zbl 089.17601.

[6] K. Dlaska and M. Ganster, Almost rc-Lindelöf spaces, Bull. Malaysian Math. Soc. (2) 17 (1997), 51-56.

[7] J. Dontchev, M. Ganster, and T. Noiri, On p-closed spaces, Int. J. Math. Math. Sci. 24 (2000), no. 3, 203-212. MR 2001c:54017. Zbl 0965.54024.

[8] N. El-Deeb, I. A. Hasanein, A. S. Mashhour, and T. Noiri, On p-regular spaces, Bull. Math. Soc. Sci. Math. R. S. Roumanie (N.S.) 27(75) (1983), no. 4, 311-315. MR 85d:54018. Zbl 524.54016.

[9] Z. Frolík, Remarks concerning the invariance of Baire spaces under mappings, Czechoslovak Math. J. 11(86) (1961), 381-385. MR 24\#A2932. Zbl 104.17204.

[10] T. Husain, Almost continuous mappings, Prace Mat. 10 (1966), 1-7. MR 36\#3322. Zbl 138.17601.

[11] S. Jafari and T. Noiri, On almost precontinuous functions, Int. J. Math. Math. Sci. 24 (2000), no. 3, 193-201. CMP 1776 323. Zbl 958.54011. 
[12] D. S. Janković, $\theta$-regular spaces, Int. J. Math. Math. Sci. 8 (1985), no. 3, 615-619. MR 87h:54030. Zbl 577.54012.

[13] N. Levine, Semi-open sets and semi-continuity in topological spaces, Amer. Math. Monthly 70 (1963), 36-41. MR 29\#4025. Zbl 113.16304.

[14] A. S. Mashhour, M. E. Abd El-Monsef, and S. N. El-Deep, On precontinuous and weak precontinuous mappings, Proc. Math. Phys. Soc. Egypt 53 (1982), 47-53. Zbl 571.54011.

[15] A. S. Mashhour, I. A. Hasanein, and S. N. El-Deeb, A note on semicontinuity and precontinuity, Indian J. Pure Appl. Math. 13 (1982), no. 10, 1119-1123. MR 84a:54021. Zbl 499.54009 .

[16] A. A. Nasef and T. Noiri, Some weak forms of almost continuity, Acta Math. Hungar. 74 (1997), no. 3, 211-219. CMP 1440 246. Zbl 924.54017.

[17] O. Njȧstad, On some classes of nearly open sets, Pacific J. Math. 15 (1965), 961-970. MR 33\#3245. Zbl 137.41903.

[18] T. Noiri, Properties of hyperconnected spaces, Acta Math. Hungar. 66 (1995), no. 1-2, $147-$ 154. MR 95k:54037. Zbl 818.54020.

[19] Strongly 9-precontinuous functions, Acta Math. Hungar. 90 (2001), 315-324.

[20] M. C. Pal and P. Bhattacharyya, Feeble and strong forms of preirresolute functions, Bull. Malaysian Math. Soc. (2) 19 (1996), no. 2, 63-75. CMP 1464 562. Zbl 885.54010.

[21] R. Paul and P. Bhattacharyya, Quasi-pre-continuous functions, J. Indian Acad. Math. 14 (1992), no. 2, 115-126. CMP 1261 772. Zbl 871.54018.

[22] V. Pipitone and G. Russo, Spazi semiconnessi e spazi semiaperti, Rend. Circ. Mat. Palermo (2) 24 (1975), no. 3, 273-285 (Italian). MR 57\#4084.

[23] V. Popa and T. Noiri, Almost weakly continuous functions, Demonstratio Math. 25 (1992), no. 1-2, 241-251. MR 93f:54020. Zbl 789.54014.

[24] Weakly $\beta$-continuous functions, An. Univ. Timişoara Ser. Mat.-Inform. 32 (1994), no. 2, 83-92. MR 97g:54019. Zbl 864.54009.

[25] J. Porter and J. Thomas, On H-closed and minimal Hausdorff spaces, Trans. Amer. Math. Soc. 138 (1969), 159-170. MR 38\#6544. Zbl 175.49501.

[26] V. Pták, Completeness and the open mapping theorem, Bull. Soc. Math. France 86 (1958), 41-74. MR 21\#4345. Zbl 082.32502.

[27] N. V. Veličko, H-closed topological spaces, Amer. Math. Soc. Transl. (2) 78 (1968), 103-118.

TAKAShi Noiri: Department of MAThematics, Yatsushiro College of Technology, YATSUSHIRO, KUMAMOTO, 866-8501, JAPAN

E-mail address: noiri@as.yatsushiro-nct.ac.jp 


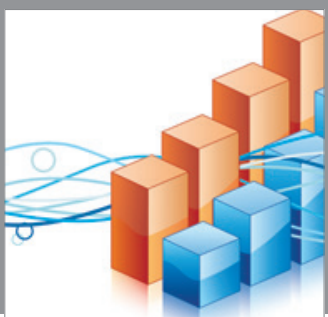

Advances in

Operations Research

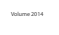

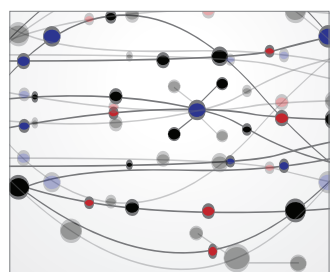

\section{The Scientific} World Journal
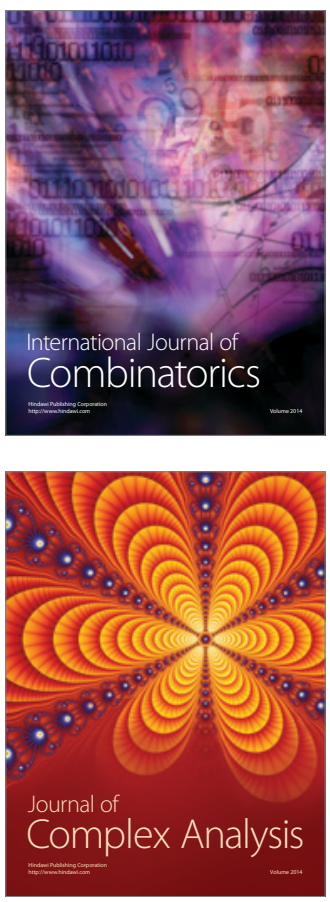

International Journal of

Mathematics and

Mathematical

Sciences
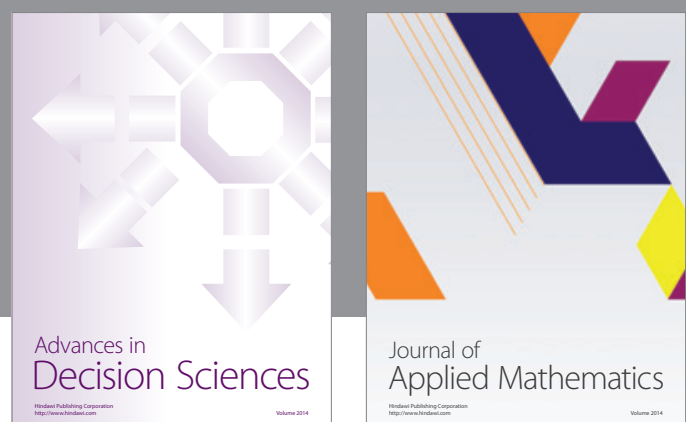

Journal of

Applied Mathematics
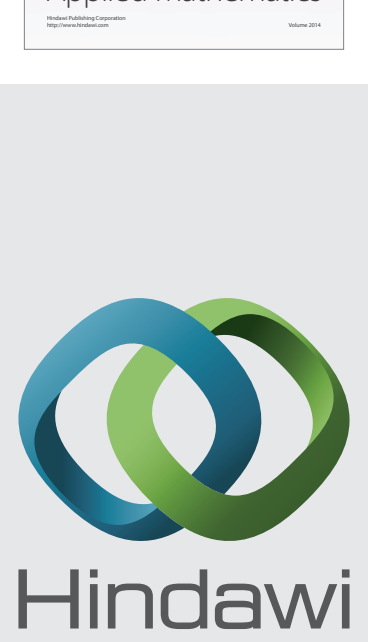

Submit your manuscripts at http://www.hindawi.com
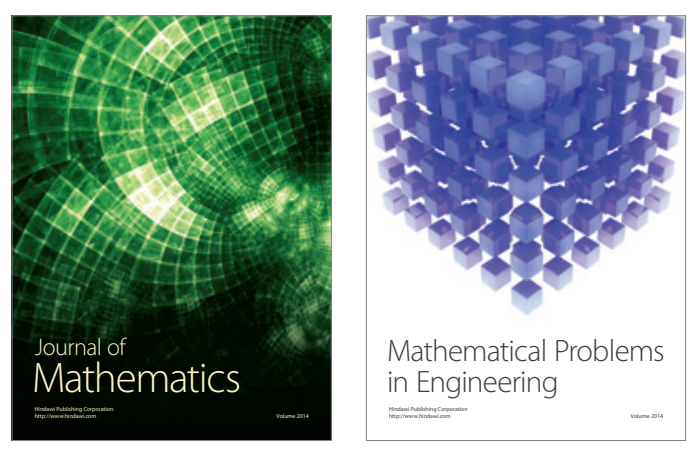

Mathematical Problems in Engineering
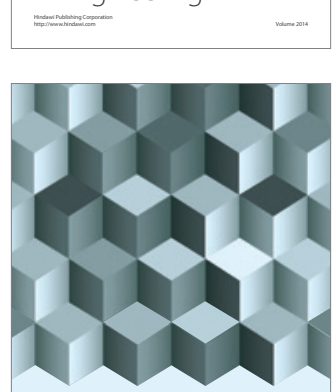

Journal of

Function Spaces
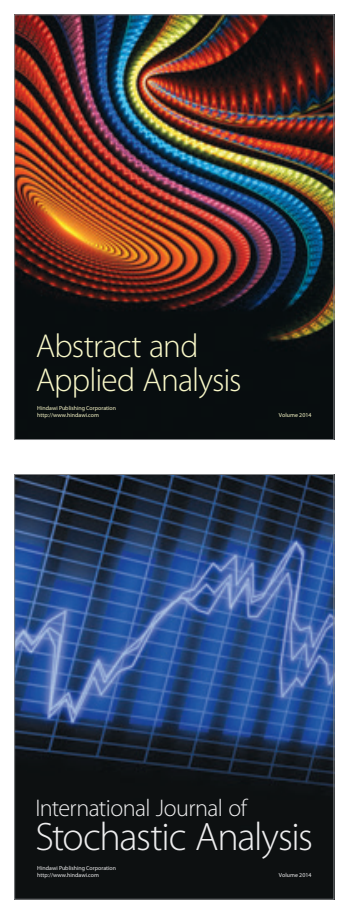

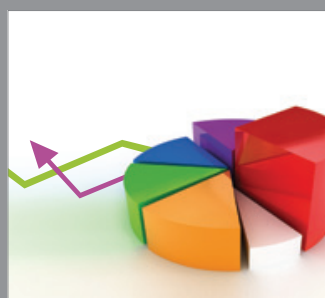

ournal of

Probability and Statistics

Promensencen
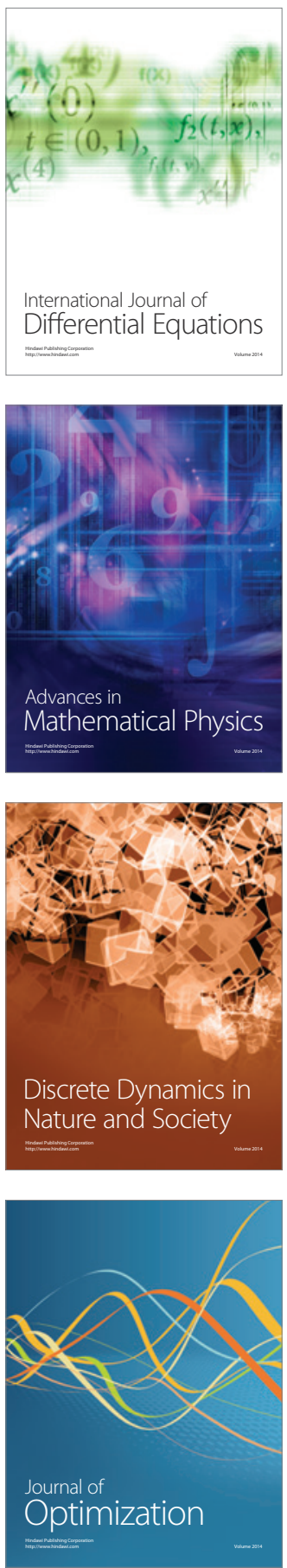